\title{
Spin-Hall Effect in a [110] GaAs Quantum Well
}

\author{
E. M. Hankiewicz and G. Vignale \\ Department of Physics and Astronomy, University of Missouri, Columbia, Missouri 65211, USA* \\ M. E. Flatté \\ Department of Physics and Astronomy, University of Iowa, Iowa City, Iowa 52242, USA
}

(Received 23 March 2006; published 27 December 2006)

\begin{abstract}
A self-consistent treatment of the spin-Hall effect requires consideration of the spin-orbit coupling and electron-impurity scattering on equal footing. This is done here for the experimentally relevant case of a [110] GaAs quantum well [Sih et al., Nature Phys. 1, 31 (2005)]. Working within the framework of the exact linear response formalism we calculate the spin-Hall conductivity including the Dresselhaus linear and cubic terms in the band structure, as well as the electron-impurity scattering and electron-electron interaction to all orders. We show that the spin-Hall conductivity naturally separates into two contributions, skew-scattering and side-jump, and we propose an experiment to distinguish between them.
\end{abstract}

DOI: 10.1103/PhysRevLett.97.266601

Introduction.-The current focus of spintronics based on spin-orbit (SO) interactions is on the spin-Hall effect [1-4] (SHE), i.e., the generation of a steady spin-current transverse to a dc electric field. The recent experimental observations of the SHE [5-7] have enhanced the interest of the community in this topic. At first sight the SHE seems to arise from two quite different mechanisms: one associated with SO interactions between electrons and impurities $[1,2,8,9]$, and the other connected with SO interactions in the band structure of the material $[3,4]$. In reality, the two mechanisms are inseparable. On one hand, the presence of electron-impurity scattering is essential to ensure that the system reaches a steady state in the presence of the electric field. This means that the dc limit $(\omega \rightarrow 0$, where $\omega$ is the frequency of the electric field) must be taken before letting the electron-impurity scattering time $\tau$ tend to infinity in the "clean limit." On the other hand, the unitary transformation that reduces the original multiband Hamiltonian of the solid to an effective Hamiltonian for, say, the conduction band, not only generates SO coupling terms (for example the Dresselhaus term) but also modifies the position operator [see Eq. (3) below]. All the above effects have an impact on the spin-Hall conductivity (SHC). The socalled skew-scattering (SS) contribution [10] arises from the asymmetry of the electron-impurity scattering in the presence of SO interactions [11]. The "side-jump" (SJ) [12-14] comes from the change in the form of the position and velocity operators. Finally, the "intrinsic" [15] contribution arises from the SO coupling terms in the effective Hamiltonian. Notice that the side-jump and the intrinsic contributions have a common origin in the unitary transformation that reduces the multiband Hamiltonian to an effective one-band Hamiltonian.

Although all types of contributions are present in experiments [5-7], theoretical approaches usually focus on one or the other. Some papers focus on the band structure effects neglecting the effect of spin-orbit coupling on electron-impurity scattering $[16,17]$, others on the SS and
PACS numbers: 72.25.Dc, 72.25.Rb, 73.43.-f, 85.75.-d

SJ effects, ignoring spin-orbit interactions in the band structure $[9,18,19]$. A complete theory should of course describe all contributions on equal footing [20]. Furthermore, it would be desirable to have a way to distinguish experimentally the various contributions.

In this Letter we present a complete theory of the SHE for an experimentally well-studied system, a GaAs [110] quantum well (QW)[7], in which the electron-impurity and the electron-electron interaction can be studied simultaneously and consistently with the spin-orbit terms in the band structure, namely, the linear and cubic Dresselhaus terms. Let us emphasize that in this system the spin current and the SHC are well defined because the $z$ component of the spin is conserved. We show that the Dresselhaus terms do not contribute while the conservation of $S_{z}$ component implies that the SHC includes only "pure" skew-scattering and side-jump contributions. Furthermore, we are able to prove (using exact linear response theory) that the sidejump contribution is independent of the strength of disorder and Coulomb interactions, a fact that had been shown on the basis of perturbative calculations to first order in disorder $[14,21,22]$, but never before shown to be true at all orders in the strength of the interactions and disorder potential. By contrast, in a [001] QW we find that the nonconservation of $S_{z}$ causes corrections to the sidejump effect, as well as the appearance of intrinsic contributions to the SHC from the nonlinear Dresselhaus term.

Thus, the spin-Hall effect in a [110] GaAs QW offers a hitherto unexplored opportunity to measure pure skewscattering and side-jump contributions. And since the skew-scattering spin-Hall conductivity increases with increasing mobility while the side-jump conductivity is not affected, we can propose a new experiment, where the change in the sign of SHC with temperature reveals the dominance of one or the other mechanisms.

Model and results. - Our effective Hamiltonian for the conduction band of a [110] QW includes Dresselhaus spinorbit couplings, as well as SO corrections to the electron- 
impurity and the electron-electron $(e-e)$ interactions:

$$
\begin{aligned}
\hat{H}= & \sum_{i=1}^{N}\left[\frac{\hat{p}_{i}^{2}}{2 m^{*}}+V\left(\hat{\vec{r}}_{i}\right)\right]+\frac{1}{2} V_{e-e} \\
& +\frac{\alpha_{1}}{\hbar} \sum_{i=1}^{N}\left(\hat{p}_{i x} \nabla_{i y} \hat{V}_{T i}-\hat{p}_{i y} \nabla_{i x} \hat{V}_{T i}\right) \hat{S}_{i z} \\
& +\sum_{i=1}^{N}\left[\frac{\alpha_{2}}{\hbar} e E_{0} \hat{p}_{i y} \hat{S}_{i z}+\frac{\alpha_{2}^{\prime}}{\hbar} e E_{0}\left(-\hat{p}_{i y} \hat{p}_{i x}^{2}+\hat{p}_{i y}^{3} / 2\right) \hat{S}_{i z}\right] .
\end{aligned}
$$

Here $\hat{\vec{p}}_{i}, \hat{\vec{r}}_{i}$ are the canonical momentum and position operators of the $i$ th electron, $V_{e-e}=\sum_{j \neq i} \frac{e^{2}}{\epsilon_{b}\left|\hat{\vec{r}}_{i}-\hat{\vec{r}}_{j}\right|}, \hat{V}_{T i} \equiv$ $V\left(\hat{\vec{r}}_{i}\right)+V_{e-e}$ is the total potential acting on the $i$ th electron due to random impurities $[V(\vec{r})]$ and $e$-e interactions in the (110) plane, $\hat{S}_{i z}$ is the Pauli matrix operator, $x, y$, and $z$ are Cartesian component indices with $z$ along the [110] axis, $m^{*}$ is the conduction band mass, $e$ is the absolute value of the electron charge, $E_{0}$ is a "built-in" electric field originating from the crystal symmetry, and the quantities $\alpha_{1}$, $\alpha_{2}, \alpha_{2}^{\prime}$ are the strengths of SO coupling in the semiconductor, whose values can be calculated from the matrix elements of the momentum operator between different bands within the $14 \times 14$ band model [23]. Notice that $\hat{S}_{i z}$ is a constant of the motion. Although $\alpha_{1}, \alpha_{2}$, and $\alpha_{2}^{\prime}$ are connected, we assign them different labels in order to (artificially) turn off one or the other effect in our calculations. These calculations are done to first order in the $\alpha$ 's, which is correct if energies associated with the $\alpha$ 's are much smaller than the Fermi energy. Notice that the SO coupling energy is not required to be much smaller than $\hbar / \tau$.

We perturb the system with a uniform electric field of frequency $\omega$ in the $x$ direction, which is described by a vector potential $\frac{e}{c} \vec{A}(t)=\frac{e}{i \omega} E \vec{e}_{x} e^{-i \omega t}+$ c.c. The perturbed Hamiltonian, to first order in $E$, is obtained from the canonical replacement $\hat{\vec{p}} \rightarrow \hat{\vec{p}}+\frac{e}{c} \vec{A}(t)$, which gives

$$
\begin{aligned}
\hat{H}(t)= & \hat{H}+\frac{e}{c} \sum_{i=1}^{N}\left(\frac{\hat{p}_{i x}}{m^{*}}-2 \frac{\alpha_{2}^{\prime}}{\hbar} e E_{0} \hat{p}_{i y} \hat{p}_{i x} \hat{S}_{i z}\right. \\
& \left.+\frac{\alpha_{1}}{\hbar} \nabla_{i y} \hat{V}_{T i} \hat{S}_{i z}\right) A_{x}(t) .
\end{aligned}
$$

We want to calculate the magnitude of the transverse $z$-spin current defined as $\hat{J}_{y}^{z} \equiv \frac{\hbar}{2} \sum_{i=1}^{N} \frac{\hat{v}_{i y} \hat{S}_{i z}+\hat{S}_{i z} \hat{v}_{i y}}{2}$, where $\hat{v}_{i y}$ is the $y$ component of the velocity operator. To find the correct expression for $\hat{\vec{v}}_{i}$ we note that, as a result of the transformation from the original multiband Hamiltonian to the effective Hamiltonian Eq. (2), the physical position operator no longer coincides with the canonical position operator $\hat{\vec{r}}_{i}$, but is given by [24]

$$
\hat{\vec{r}}_{\mathrm{phys}, i}=\hat{\vec{r}}_{i}-\frac{\alpha_{1}}{\hbar}\left(\hat{\vec{p}}_{i}+\frac{e}{c} \vec{A}\right) \times \hat{\vec{S}}_{i} .
$$

The velocity is the time derivative of $\hat{\vec{r}}_{\text {phys }}$ and making use of $-\frac{1}{c} \dot{\vec{A}}=\vec{E}$ we find (to first order in the $\alpha$ 's and dropping a "diamagnetic" term with zero average)

$$
\begin{aligned}
\hat{v}_{i y}= & \frac{1}{m^{*}} \hat{p}_{i y}-\frac{\alpha_{1}}{\hbar} \nabla_{i x} \hat{V}_{T i} \hat{S}_{i z} \\
& +\left[\frac{\alpha_{2}}{\hbar} e E_{0}+\frac{\alpha_{2}^{\prime}}{\hbar} e E_{0}\left(-\hat{p}_{i x}^{2}+\frac{3 \hat{p}_{i y}^{2}}{2}\right)\right] \hat{S}_{i z} \\
& -\frac{\alpha_{1}}{\hbar} \nabla_{i x} \hat{V}_{T i} \hat{S}_{i z}-\frac{\alpha_{1} e}{\hbar} E \hat{S}_{i z} .
\end{aligned}
$$

It is essential for what follows that a steady state be established in response to the dc electric field. In the steady state regime the average force exerted on electrons of either spin by impurities and other electrons $\left\langle-\vec{\nabla} V_{T}\right\rangle$ must be exactly balanced by the average force exerted by the electric field $-e \vec{E}$. So for the average spin current, the last two terms of Eq. (4) cancel out and we get

$$
\begin{aligned}
J_{y}^{z}= & \frac{\hbar}{2}\left\{\frac{\alpha_{1} n e}{\hbar} E+\frac{\alpha_{2} n e}{\hbar} E_{0}\right. \\
& \left.+\sum_{i=1}^{N}\left[\frac{\hat{p}_{i y}}{m} \hat{S}_{i z}+\frac{\alpha_{2}^{\prime}}{\hbar} e E_{0}\left(-\hat{p}_{i x}^{2}+\frac{3}{2} \hat{p}_{i y}^{2}\right)\right]\right\},
\end{aligned}
$$

where $n$ is the electron density. The first term on the righthand side (rhs) of Eq. (5) produces one half of the sidejump contribution to the SHC, while the second one is the intrinsic term generated by the internal field $E_{0}$. The SJ and intrinsic terms have the same form. However, the term $\alpha_{2} n e E_{0} / \hbar$ does not depend on the external electric field and because $\left\langle J_{y}^{z}\right\rangle=0$ in the ground-state, it is canceled by the ground-state average of the other terms.

Now we use the linear response theory to calculate the average spin current $\left\langle J_{y}^{z}\right\rangle$ to first order in $E$. So we write $J_{y}^{z}(t)=\sigma_{y x}^{\mathrm{SH}}(\omega) E e^{-i \omega t}+$ c.c., where the spin-Hall conductivity, $\sigma_{y x}^{\mathrm{SH}}(\omega)$ to the first order in the strength of SO coupling is given by the Kubo formula:

$$
\begin{aligned}
\sigma_{y x}^{\mathrm{SH}}(\omega)= & \frac{1}{2} \alpha_{1} n e \\
& +\frac{e \hbar}{2 i \omega \mathcal{A}}\left[\frac{\alpha_{1}}{\hbar}\left\langle\left\langle\sum_{i=1}^{N} \frac{\hat{p}_{i y} \hat{S}_{i z}}{m^{*}} ; \sum_{i=1}^{N} \nabla_{i y} \hat{V}_{T i} \hat{S}_{i z}\right\rangle\right\rangle\right. \\
& +\left\langle\left\langle\sum_{i=1}^{N} \frac{\hat{p}_{i y} \hat{S}_{i z}}{m^{*}} ; \frac{\hat{P}_{x}}{m^{*}}+2 \frac{\alpha_{2}^{\prime}}{\hbar} e E_{0} \sum_{i=1}^{N} \hat{p}_{i y} \hat{p}_{i x} \hat{S}_{i z}\right\rangle\right\rangle \\
& \left.+\frac{\alpha_{2}^{\prime}}{\hbar}\left\langle\left\langle e E_{0} \sum_{i=1}^{N}\left(-\hat{p}_{i x}^{2}+\frac{3}{2} \hat{p}_{i y}^{2}\right) ; \frac{\hat{P}_{x}}{m^{*}}\right\rangle\right\rangle\right]
\end{aligned}
$$

where $\hat{\vec{P}}$ is the total momentum operator of the system, $\mathcal{A}$ is the area of the 2DEG, and $\langle\langle\hat{A} ; \hat{B}\rangle\rangle_{\omega} \equiv-\frac{i}{\hbar} \times$ $\int_{0}^{\infty}\langle[\hat{A}(t), \hat{B}(0)]\rangle e^{-i \omega t} d t$, where $\langle\ldots\rangle$ is shorthand for the Kubo linear response function.

We now show that the second term on the rhs of Eq. (6) can be calculated exactly and, combined with the first term, gives the full side-jump contribution to the SHC. We first 
note that according to the Heisenberg equation of motion for the momentum operator, to zero order in $\alpha_{1}$ we can write $\nabla_{i y} \hat{V}_{T i}=-\frac{d}{d t} \hat{p}_{i y}$. Furthermore $\hat{S}_{i z}$ is a strict constant of the motion, so we also have $\nabla_{i y} \hat{V}_{T i} \hat{S}_{i z}=-\frac{d}{d t}\left(\hat{p}_{i y} \hat{S}_{i z}\right)$. Making use of this identity the term in question can be rewritten as

$$
-\frac{e \alpha_{1}}{2 i \omega \mathcal{A}}\left\langle\left\langle\sum_{i=1}^{N} \frac{\hat{p}_{i y} \hat{S}_{i z}}{m^{*}} ; \sum_{i=1}^{N} \frac{d}{d t}\left(\hat{p}_{i y} \hat{S}_{i z}\right)\right\rangle\right\rangle=-\frac{\alpha_{1} e}{2 m^{*}}\left\langle\left\langle\hat{P}_{y} ; \hat{P}_{y}\right\rangle\right\rangle
$$

where in the last step we have used the well-known property of linear response functions,

$$
\langle\langle\hat{A} ; \hat{B}\rangle\rangle_{\omega}=-\frac{\langle\langle d \hat{A} / d t ; \hat{B}\rangle\rangle_{\omega}}{i \omega}+\frac{\langle[\hat{A}, \hat{B}]\rangle}{\omega},
$$

and the fact that $\hat{S}_{i z}^{2}=1$.

The value of the $\left\langle\left\langle\hat{P}_{y} ; \hat{P}_{y}\right\rangle\right\rangle$ response function in the $\omega \rightarrow$ 0 limit is easily obtained from the condition that the ordinary de conductivity

$$
\sigma_{y y}(\omega)=\frac{-1}{i \mathcal{A} \omega}\left[\left\langle\left\langle-e \frac{\hat{P}_{y}}{m^{*}} ;-e \frac{\hat{P}_{y}}{m^{*}}\right\rangle\right\rangle_{\omega}+\frac{N e^{2}}{m^{*}}\right]
$$

is finite for $\omega \rightarrow 0$ [25]. This implies that the quantity in the square brackets of Eq. (9) vanishes in the limit $\omega \rightarrow 0$, i.e., $[26,27]$ :

$$
\lim _{\omega \rightarrow 0}\left\langle\left\langle\hat{P}_{y} ; \hat{P}_{y}\right\rangle\right\rangle=-N m^{*} .
$$

Furthermore, it is easy to see that the terms proportional to $\alpha_{2}^{\prime}$ in the last two lines of Eq. (6) vanish for $\alpha_{1}=0$ (this is a consequence of the fact that for $\alpha_{1}=0$ these terms are odd functions of $p_{i x}$ while the Hamiltonian is an even function of $p_{i x}$ ): hence they are at least of order $\alpha_{2}^{\prime} \alpha_{1}$ and can be safely neglected. Then Eq. (6), in the de limit, simplifies to

$$
\sigma_{y x}^{\mathrm{SH}}=\alpha_{1} n e+\lim _{\omega \rightarrow 0} \frac{e \hbar}{2 i \omega \mathcal{A}}\left\langle\left\langle\sum_{i=1}^{N} \frac{\hat{p}_{i y} \hat{S}_{i z}}{m^{*}} ; \frac{\hat{P}_{x}}{m^{*}}\right\rangle\right\rangle .
$$

The first and the second term on the rhs of this equation are naturally identified as the side-jump and the skewscattering contributions to the SHC. Indeed the second term on the rhs of Eq. (11) is simply the response of the $y$ component of the canonical spin current to an electric field that couples to the $x$ component of the canonical particle current. This response includes neither the anomalous velocity nor the anomalous coupling to the electric field, and furthermore it vanishes for $\alpha_{1}=0$ (again, because of odd parity with respect to $p_{i x}$ ); hence, it cannot be sustained by the band structure alone.

Thus, we conclude that the total SHC for a [110] QW [7] is the sum of a universal side-jump contribution and a skew-scattering contribution due to the impurities:

$$
\sigma_{y x}^{\mathrm{SH}}=\sigma_{y x}^{s j}+\sigma_{y x}^{s s},
$$

where

$$
\sigma_{y x}^{s s}=\lim _{\omega \rightarrow 0} \frac{e \hbar}{2 i \omega \mathcal{A}}\left\langle\left\langle\sum_{i=1}^{N} \frac{\hat{p}_{i y} \hat{S}_{i z}}{m^{*}} ; \frac{\hat{P}_{x}}{m^{*}}\right\rangle\right\rangle
$$

and $\sigma_{y x}^{s j}=\alpha_{1} n e$.
Notice that we have not assumed that either disorder or the Coulomb interaction is weak: thus we have proved that the side-jump SHC in a [110] QW is independent of disorder and the Coulomb potential to all orders in the strength of these interactions. The proof, however, depends on the conservation of $\hat{S}_{z}$, which is a special feature of the [110] QW. When $\hat{S}_{z}$ is not conserved, then Eq. (7) is no longer true. The spin precessional frequency $\Omega_{p}$, while proportional to $\alpha$, will not be small in comparison to $1 / \tau$ in the clean limit. As a result, terms that could be safely disregarded when $\Omega_{p}$ was zero (conserved spin), become very large when $\Omega_{p} \tau \gg 1$ and may even diverge when $1 / \tau$ tends to zero before $\Omega_{p}$. This is the basic mechanism through which the side-jump contribution can be modified by spin precession and intrinsic contributions to the SHC can also appear. Indeed, in the case of a [001] GaAs QW we find that the left-hand side of Eq. (7) vanishes in the clean limit, thus reducing the side-jump contribution to $\frac{1}{2} \alpha_{1} n e$.

Let us now return to the [110] QW. The skew-scattering contribution [Eq. (13)] is not easily obtained from perturbation theory, but we have recently shown, via the Boltzmann equation, that it is given by

$$
\sigma_{y x}^{s s}=-\mu \frac{\hbar n \tau / \tau_{s s}}{1+\gamma \tau},
$$

where $\mu$ is the mobility, $\tau_{s s}$ is the SS relaxation time inversely proportional to $\alpha_{1}$, and $\gamma$ is the spin-drag coefficient $[9,28,29]$. It is seen that $e-e$ interactions are quite relevant here and reduce the SS term in Eq. (14) by the factor $1+\gamma \tau$. Moreover, the two contributions in Eq. (12) have opposite signs for an attractive impurity potential, typical for mobilities dominated by the dopants producing mobile carriers, and the SS conductivity increases with the mobility while the SJ is independent of it. If the mobility is dominated by repulsive impurity potentials the two contributions in Eq. (12) have the same sign [18]. Similarly, the spin accumulation consists of two terms with opposite signs and has the form [9]: $V_{\mathrm{ac}}(W / 2)=$ $-2 L_{s} j_{x} \rho_{D}\left(\tau / \tau_{s s}-2 e \alpha_{1} / \hbar \mu\right) \tanh \left(W / 2 L_{s}\right)$, where $\rho_{D}$ is the Drude resistivity, $L_{s}$ is the spin diffusion length, $W$ is the width of sample, and $j_{x}$ is the current density [30]. We then propose a new experiment where the SJ/SS contributions can be distinguished through the temperature dependence of $\sigma_{y x}^{\mathrm{SH}}$ or the spin accumulation potential $V_{\mathrm{ac}}$. Figure 1 presents the behavior of $\mu$ versus $T$ for experimentally attainable samples. Notice that the values of parameters for theoretical curve designated by circles are exactly the same as the values reported for the samples in 


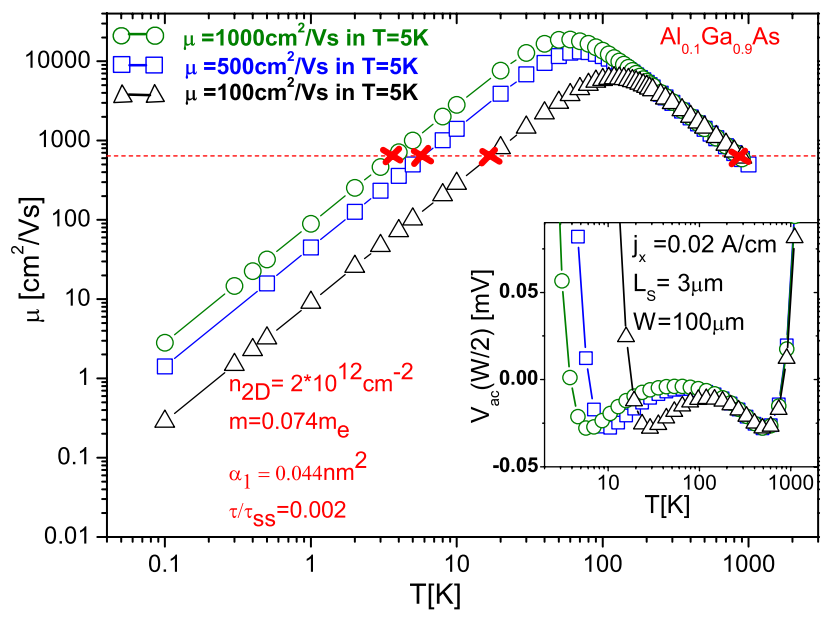

FIG. 1 (color online). Mobility $\mu$, as a function of temperature $T$, for three different low- $T \mu$ 's. In the inset, the spin accumulation $\left(V_{\text {ac }}\right)$ vs $T$. The side-jump contribution to $V_{\text {ac }}$ dominates for low $T$. For increasing $T$, the lower temperature red (or dark gray) cross corresponds to the $T$ where the sign of $V_{\text {ac }}$ starts to be controlled by skew scattering, the higher temperature red (or dark gray) cross to the place where side jump dominates again.

the recent experiments [7] on a [110] QW. The samples with lower mobilities can be easily obtained by additional doping with $\mathrm{Si}$ inside the quantum well [31]. Hence $\mu$ will grow as $T^{3 / 2}$ for low $T$ as a result of scattering from ionized impurities and will decrease as $T^{-3 / 2}$ for larger $T$ due to phonon scattering. It is thus possible to observe two changes of sign of $V_{\mathrm{ac}}$ moving from low to high $T \mathrm{~s}: \mu=$ $1 /\left(A T^{-3 / 2}+B T^{3 / 2}\right)$, where $A$ is found from the low- $T$ mobility and $B$ is fixed by a room temperature mobility of $0.3 \mathrm{~m}^{2} / \mathrm{Vs}$ for AlGaAs. At low $T$ the mobility is low and the $\mathrm{SJ}$ contribution to $V_{\mathrm{ac}}$ dominates. The first cross in the graph designates the point where the SS begins to dominate, and the second cross, at still higher $T$, is the point where the SJ retakes control of the sign of $V_{\mathrm{ac}}$. Even if the sign change is not detected, it is possible to tell whether SS or SJ dominates by measuring whether $V_{\text {ac }}$ increases or decreases as $\mu$ increases with changing $T$.

Summary. - We have studied the spin-Hall effect for a [110] QW taking into account the Dresselhaus SO coupling terms and the spin-orbit interaction between electrons and impurities in the presence of electron-electron interactions. We have shown that in the recent experiment of Ref. [7] the spin-Hall effect presents a clean competition between sidejump and skew-scattering contributions (no intrinsic terms). Furthermore, we have proposed a new experiment where the change in the sign of spin-Hall accumulation with temperature reveals the dominance of one or the other effect. Finally, we have proved that the side-jump part of the spin-Hall conductivity is independent of disorder and the Coulomb interaction at all orders, provided the spin current is associated with a conserved component of the spin.
We thank Jairo Sinova for useful comments. This work was supported by NSF Grant No. DMR-0313681, DARPA/ ONR N00014-05-1-0913, an ONR MURI, and NSF Grant No. PHY99-07949.

*Electronic address: hankiewicze@missouri.edu

[1] M. I. Dyakonov and V.I. Perel, Phys. Lett. A 35, 459 (1971).

[2] J. E. Hirsch, Phys. Rev. Lett. 83, 1834 (1999).

[3] S. Murakami, N. Nagaosa, and S.-C. Zhang, Science 301, 1348 (2003).

[4] J. Sinova et al., Phys. Rev. Lett. 92, 126603 (2004).

[5] Y. K. Kato et al., Science 306, 1910 (2004).

[6] J. Wunderlich et al., Phys. Rev. Lett. 94, 047204 (2005).

[7] V. Sih et al., Nature Phys. 1, 31 (2005).

[8] S. Zhang, Phys. Rev. Lett. 85, 393 (2000).

[9] E. M. Hankiewicz and G. Vignale, Phys. Rev. B 73, 115339 (2006).

[10] J. Smit, Physica (Utrecht) 21, 877 (1955); 24, 39 (1958).

[11] N.F. Mott and H.S. W. Massey, The Theory of Atomic Collisions (Oxford University Press, New York, 1964).

[12] L. Berger Phys. Rev. B 2, 4559 (1970); 5, 1862 (1972).

[13] S. K. Lyo and T. Holstein, Phys. Rev. Lett. 29, 423 (1972).

[14] P. Noziéres and C. Lewiner, J. Phys. (Paris) 34, 901 (1973).

[15] H. A. Engel, E. Rashba, and B. I. Halperin, cond-mat/ 0603306.

[16] J. I. Inoue, G. E. W. Bauer, and L. W. Molenkamp, Phys. Rev. B 70, 041303(R) (2004).

[17] E. G. Mishchenko, A. V. Shytov, and B. I. Halperin, Phys. Rev. Lett. 93, 226602 (2004).

[18] H. A. Engel, B. I. Halperin, and E. Rashba, Phys. Rev. Lett. 95, 166605 (2005).

[19] W. K. Tse and S. D. Sarma, Phys. Rev. Lett. 96, 056601 (2006).

[20] W. K. Tse and S.D. Sarma, cond-mat/0602607 [Phys. Rev. B (to be published)].

[21] J. M. Luttinger, Phys. Rev. 112, 739 (1958).

[22] A. Crépieux and P. Bruno, Phys. Rev. B 64, 014416 (2001).

[23] R. Winkler, Spin-Orbit Effects in Two-Dimensional Electron and Hole Systems (Springer, New York, 2003).

[24] For an eight band model the formula for the position operator was derived in Ref. [14]. We found that the same formula is valid for 14 band model.

[25] It is in this step that we assume the system to be metallic.

[26] Since, by translational invariance, $\left\langle\left\langle\hat{P}_{y} ; \hat{P}_{y}\right\rangle\right\rangle_{\omega}=0$ in a perfectly clean system at any finite frequency, such a cancellation can occur only in the presence of disorder.

[27] This result is exact in the absence of spin-orbit couplings. Spin-orbit effects introduce negligible correction of the order $E_{\mathrm{so}} / E_{F}$, where $E_{\mathrm{so}}$ is the characteristic energy of spin-orbit interaction and $E_{F}$ is the Fermi energy.

[28] I. D'Amico and G. Vignale, Phys. Rev. B 62, 4853 (2000).

[29] C. P. Weber et al., Nature (London) 437, 1330 (2005).

[30] We omitted the spin Coulomb drag correction which is small for the mobilities considered here.

[31] D. Awschalom (private communication). 